\title{
Statistical precision of the intensities retrieved from constrained fitting of overlapping peaks in high-resolution mass spectra
}

\author{
M. J. Cubison ${ }^{1,2}$ and J. L. Jimenez ${ }^{1}$ \\ ${ }^{1}$ CIRES and Dept. of Chemistry and Biochemistry, University of Colorado, Boulder, CO, USA \\ ${ }^{2}$ TOFWERK AG, Thun, Switzerland
}

Correspondence to: M. J. Cubison (cubison@ tofwerk.com)

Received: 24 October 2014 - Published in Atmos. Meas. Tech. Discuss.: 17 December 2014

Revised: 20 April 2015 - Accepted: 10 May 2015 - Published: 5 June 2015

\begin{abstract}
Least-squares fitting of overlapping peaks is often needed to separately quantify ions in high-resolution mass spectrometer data. A statistical simulation approach is used to assess the statistical precision of the retrieved peak intensities. The sensitivity of the fitted peak intensities to statistical noise due to ion counting is probed for synthetic data systems consisting of two overlapping ion peaks whose positions are pre-defined and fixed in the fitting procedure. The fitted intensities are sensitive to imperfections in the $m / Q$ calibration. These propagate as a limiting precision in the fitted intensities that may greatly exceed the precision arising from counting statistics. The precision on the fitted peak intensity falls into one of three regimes. In the "countinglimited regime" (regime I), above a peak separation $\chi \sim 2$ to 3 half-widths at half-maximum (HWHM), the intensity precision is similar to that due to counting error for an isolated ion. For smaller $\chi$ and higher ion counts ( $\sim 1000$ and higher), the intensity precision rapidly degrades as the peak separation is reduced ("calibration-limited regime", regime II). Alternatively for $\chi<1.6$ but lower ion counts (e.g. 10-100) the intensity precision is dominated by the additional ion count noise from the overlapping ion and is not affected by the imprecision in the $m / Q$ calibration ("overlapping-limited regime", regime III). The transition between the counting and $m / Q$ calibration-limited regimes is shown to be weakly dependent on resolving power and data spacing and can thus be approximated by a simple parameterisation based only on peak intensity ratios and separation. A simple equation can be used to find potentially problematic ion pairs when evaluating results from fitted spectra containing many ions. Longer integration times can improve the precision in regimes I and III, but a given ion pair can only be moved
\end{abstract}

out of regime II through increased spectrometer resolving power. Studies presenting data obtained from least-squares fitting procedures applied to mass spectral peaks should explicitly consider these limits on statistical precision.

\section{Introduction}

Spectra acquired using techniques such as mass spectrometry (MS) can contain large amounts of information but are inherently complex in nature and can represent a significant challenge for data analysis. The identification and separate quantification of overlapping peaks in measured spectra are often required in order to extract the maximum possible information content. Computational approaches to this deconvolution problem have been extensively reported in the literature, in both mass spectroscopy fields such as liquidchromatography MS (LC-MS; see e.g. Jaitly et al., 2009; Yu and Peng, 2010), matrix-assisted laser desorption/ionisation MS (MALDI-MS; see e.g. Sun et al., 2010; House et al., 2011), proton transfer reaction MS (PTR-MS; see e.g. Titzmann et al., 2010), electrospray ionisation MS (ESI-MS; see e.g. Horn et al., 2000; Strittmatter et al., 2003) and other techniques with similar analysis procedures such as chromatography (see e.g. Fraga and Corley, 2005; Krupcik et al., 2005) and gamma-ray spectroscopy (Hammed et al., 1993; Uher et al., 2010; Gardner et al., 2011). Assessing the precision in the fitting parameters resulting from such deconvolution procedures is important to demonstrate the reliability of the technique and understand the information content of the retrieved data (Hammed et al., 1993). However the quantification of this precision is not always discussed in the literature. 
The primary goal of many mass spectrometry applications is the correct identification and quantification of ions present in the mass spectrum. Several studies probe the sensitivity of deconvolution algorithms to perturbations in the measurement parameters by applying them to synthetic data (Laeven and Smit, 1985; Blom, 1998; Lee and Marshall, 2000; Sun et al., 2010; Hilmer and Bothner, 2011; Müller et al., 2011). Some studies are also concerned with quantifying the overlapping ion signals, in fields such as proteomics (Link et al., 1999; Mirgorodskaya et al., 2000; Bantscheff et al., 2007, 2012) and atmospheric science (DeCarlo et al., 2006; Titzmann et al., 2010; Müller et al., 2011; Jokinen et al., 2012; Yatavelli et al., 2012). Quantification of such ion signals is difficult and may be confounded by unconstrained peak position parameters, or through the use of falsely constrained peak centroids arising from an automated peak-finding algorithm. The peak intensity and position parameters and their precisions are clearly not independent. The quantification process is thus complex, and assessing the precision of the retrieved intensities is difficult.

Correct identification of unknowns below the limit where two overlapping but non-coincident peaks no longer maintain an inflection point in the derivatives of the measurement profile is difficult. However, effects such as peak width broadening may point to the presence of unknown ions. For example, Meija and Caruso (2004) use peak width measurements from a calibration standard to compare with that of a spectrum containing two overlapping peaks, showing that Gaussian deconvolution as well as shifts in the peak centroid position can be used to predict the ratio of the intensities of the ions. Blom (1998) considers the impact of a weak overlapping interference on two quantities describing peak shape, variance and skew. That study shows that deviations in the peak shape can point to the presence of an unknown interfering peak at separations well below those which would be required to separate it visually. However, Blom also concluded that an interfering peak with relative abundances of only a few percent could cause significant shifts (of a few ppm) in the centroid $m / Q$ position, even though analysis of the peak shape would not point to the presence of the unknown peak in the spectrum.

Given the challenges encountered by such studies to correctly identify unknown peaks in the MS, it is unsurprising that the uncertainties arising during peak identification are often expressed simply by confidence metrics, such as mass accuracy/error and relative ion abundance as compared to theoretical isotope patterns (e.g. Kilgour et al., 2012) rather than, as would generally be preferred, reporting the estimated precision of the fitted intensities.

Similar confidence metrics are also reported for studies attempting to quantify the intensity of known overlapping peaks. Haimi et al. (2006) qualitatively split fits into reliable and unreliable categories by comparing peak ratios for successive measurements at different concentrations. Fits were considered reliable for a standard deviation in the peak ra- tio $<25 \%$ for eight successive measurements, an arbitrary but consistent metric. This is a useful guide when interpreting experimental results but does not address the intensity precision in a quantitative manner, limiting the scope of applicability. Müller et al. (2011) reported on a more systematic approach to quantify the expected attainable precision of the peak intensity for an example synthesised system subject to counting and estimated calibration errors. Their approach was however not extended from a single example to the general case. A generalised metric to describe the performance of such deconvolution procedures is desired.

This study aims to present a quantitative, systematic analysis of the statistical precisions arising during the deconvolution of overlapping peaks for the special case where the peak positions are known a priori and held fixed in the fitting procedure. This technique is widely employed by the atmospheric-science community during analysis of data from field and also laboratory instrumentation (e.g. Farmer and Jimenez, 2010), for example the high-resolution time-offlight aerosol mass spectrometer (HR-ToF-AMS; DeCarlo et al., 2006), the proton transfer reaction time-of-flight mass spectrometer (Cappellin et al., 2009, 2011; Müller et al., 2011), the atmospheric pressure interface time-of-flight mass spectrometer (APi-TOF; Junninen et al., 2010) and the highresolution time-of-flight chemical-ionisation mass spectrometer (HRToF-CIMS; Jokinen et al., 2012; Yatavelli et al., 2012). The ionisation techniques used in the instruments ionise and fragment the molecules in a very consistent manner. Thus, one degree of freedom can often be removed from the ion fitting procedure, which is then based upon a comprehensive list of ions and their exact $m / Q$ that define the fitted centroid values. Although analysis of the measurements from the above instrumentation motivated this study, the techniques and conclusions are directly applicable to other mass spectrometry or even optical photon-counting systems, as they are based on the spectra only and make no further assumptions about the instrumental details.

Imprecision in such a constrained fitting procedure may arise from (i) noise in the measurement distribution, particularly from counting statistics of the ions of interest; (ii) the $m / Q$ calibration, which is limited itself by statistical noise on the reference ions used to determine it; (iii) the discrete nature of the measurements, i.e. the spacing of the discrete measurement points; and (iv) the instrument transfer function ("peak shape"), errors in the determination of which propagate directly into retrieval of ion signals from the fits yet must still be empirically determined and thus will contain errors. The influence of this spacing on peak fitting results is discussed in detail in Hilmer and Bother (2010). In this work, the limitations of the fitting procedure are explored with respect to measurement noise and imperfections in the $m / Q$ calibration, i.e. items $\mathrm{i}-\mathrm{ii}$ above. Item iii is briefly discussed. Since the errors from iv are not included, our results represent a best-case scenario for the precision of the retrieved parameters. 
Müller et al. (2011) conducted an error analysis on such a constrained hypothetical system using a peak model and specifications for a typical lower-resolution TOF spectrometer used (amongst other fields) in atmospheric science, and they demonstrated that the precision in the fitted peak intensities is sensitive to the ratio of the peak intensities. The precision with which the less-intense peak intensity can be retrieved becomes extremely poor for peak separations less than the full-width at half-maximum (FWHM). Müller et al. (2011) also concluded that a precise analysis could only be performed for well-separated peaks. We extend this analysis from a single example to the general case for a wide range of measured intensities, separations and resolving powers (peak widths). We investigate the relationship between peak separation and achievable peak intensity precision, and develop a parameterisation to quantify the latter.

\section{Methods}

A synthetic measurement distribution was constructed consisting of one or two Gaussian peaks of known width and centroid position. Unless stated otherwise, the synthetic peaks were generated for a fixed ion time-of-flight (iToF) resolving power $t / \Delta t=2000$ and a sample interval that gives approximately 10 discrete measurement points within 2 fullwidths at half-maximum of the peak centre. This could be obtained for example with 0.2 ns data-acquisition point spacing at $2000 \mathrm{~ns}$ (as in this study), or $1 \mathrm{~ns}$ spacing at $10000 \mathrm{~ns}$. These metrics were chosen to correspond to realistic hardware specifications for current mass spectrometer and dataacquisition systems. Figure S1 in the Supplement shows an example of a peak using these specifications with a centroid flight time of $2000 \mathrm{~ns}$ and hence peak width $1 \mathrm{~ns}$. The peak position and width parameters are given as function of iToF rather than $m / Q$, for the mass axis goes as the square root of $\mathrm{iToF}$ and a perfect Gaussian shape observed in $\mathrm{iToF}$ does not maintain its symmetry in $m / Q$. The fits are thus conducted in $\mathrm{iToF}$, the axis in which the measurement is taken. Translation to the normally specified spectrometer mass resolving power in $m / Q$ space is given simply by $m / \Delta m=t / 2 \Delta t$, and thus the mass resolving power in our default case is 1000 . From here on we refer to the mass resolving power unless otherwise stated.

To address counting error (item $i$ above), the synthetic measurement distribution was degraded, point by point, with Poisson-distributed error of magnitude $\operatorname{sqrt}(N)$ (where $N$ is the number of ions counted during the spectrum acquisition). In time-of-flight mass spectrometry, $\mathrm{iToF}$ is converted to $m / Q$ space by fitting a function, usually proportional to the square root of iToF, which is determined by fitting isolated ions of known $m / Q$. In this work we use the equation $\mathrm{iToF}=A+B \cdot \operatorname{sqrt}(m / Q)$, where $A$ and $B$ are constants. To simulate the propagation of uncertainty of this calibration into the fitting procedure (item ii above), the constrained cen- troid values in the fits were also additionally perturbed during each fit by a randomly assigned value from a Gaussian distribution of appropriate standard deviation, as discussed below. For each iteration of the fitting procedure, this perturbation was applied consistently to both fitted peaks (i.e. the calibration parameters remain equal for all peaks). However, the perturbation applied varied from one iteration to the next.

The peak shape model (item iv) was removed as a degree of freedom by utilising Gaussian shapes to represent the instrumental peak shape; the influence of the peak shape on fitted parameters is difficult to assess (Yu and Peng, 2010, and references therein) and is thus not considered here, although its relative impact should be the focus of future studies. The separation of the discrete data points (item iii) is held fixed unless otherwise noted. Further sources of uncertainty in the measurement distribution such as electronic baseline noise are not considered. In modern data-acquisition systems they are typically small compared to ion counting noise which leads to signal degradation and a non-zero mass-spectrum baseline.

After application of the Poisson-distributed noise and of the $m / Q$ calibration errors, least-squares Gaussian peak fits were applied to the measurement distribution, where only the peak intensity parameters were determined. This procedure was repeated for 10000 randomly generated cases, resulting in a histogram of the fitted peak intensities. The width of the histogram, reported as the standard deviation of a Gaussian curve fitted to the histogram distribution, thus expresses the precision with which the peak intensities can be fit.

For the case of a system with two overlapping peaks, we define a normalised separation parameter $\chi=\mathrm{d} t$ / HWHM, where $\mathrm{d} t$ is the separation of the known peak positions in iToF space and HWHM is the half-width-half-maximum of the peak (i.e. $\chi=2$ is the case where the peaks are separated by 1 FWHM).

\section{Results and discussion}

\subsection{Precision of retrieved signal intensities due to ion counting error for an isolated ion with perfect $m / Q$ calibration}

Precision theory offers a calculable method to describe the best precision with which the peak intensity of an isolated ion can be retrieved from a discrete spectrum with Poissondistributed noise (Lee and Marshall, 2000). Lee and Marshall ran simulations of least-squares fits to Gaussian peak shapes and were able to demonstrate the application of precision theory to mass spectra, giving the relationships for the standard deviations in fitted peak amplitude, $S_{\mathrm{A}}$, and centroid iToF position, $S_{\mathrm{t}}$ : 


$$
\begin{gathered}
S_{\mathrm{A}}=c_{\mathrm{A}} \sqrt{\frac{A \delta_{t}}{W}}, \\
S_{\mathrm{t}}=c_{\mathrm{t}} \sqrt{\frac{W \delta_{t}}{A}},
\end{gathered}
$$

where $A$ is the peak amplitude in counts, $W$ the FWHM of the peak, $\delta_{\mathrm{t}}$ the separation of the discrete data points and $c_{\mathrm{A}}$ and $c_{\mathrm{t}}$ are constants. It is noted that $\delta_{\mathrm{t}}$ is not varied in our study. This result is used as the starting point for the analyses in this work, and a simulation of this simple system is demonstrated in Fig. 1. Histograms are shown for the distribution of the normalised deviation in the retrieved intensity, $\Delta=\left(I_{\text {fitted }}-I_{\text {true }}\right) / I_{\text {true }}$, over 10000 fits of an isolated ion for logarithmic steps in the peak intensity $I_{\text {true }}$. The peak position and shape are known a priori, and the observed width of the histogram, $\sigma_{N}$, thus represents the precision in $\Delta$ arising purely from counting error on an isolated ion. $\sigma_{N}$ is inversely proportional to the square root of the number of ions detected $(1 / \sqrt{N})$, as predicted by the Lee and Marshall equation and shown in the inset. The minimum attainable precision is that arising from counting error, $\sigma_{N}$ (i.e. $\sigma_{I} \geq \sigma_{N}$, where $\sigma_{I}$ is the width owing to all sources of error). Figure 1 thus represents the best-case scenario for the system, in the absence of other contributing sources of uncertainty. For the example system of $I_{\text {true }}=1000$ counts, centroid iToF $t_{0}=2000 \mathrm{~ns}, \delta_{\mathrm{t}}=0.2 \mathrm{~ns}$ and $W=1 \mathrm{~ns}$ (i.e. resolving power of 1000, as shown in Fig. S1), the precision on the retrieved intensity owing purely to Poisson-distributed counting error is thus $\sigma_{N}=3.2 \%$. Regardless of data-point spacing or resolving power, this value of $\sigma_{N}$ remains fixed for this peak intensity, since the dependencies of $A, W$ and $\delta_{\mathrm{t}}$ upon one another cancel out. For example, doubling data-acquisition rate halves the peak amplitude for the same total number of ions (ignoring non-linear effects from the detector which are outside the scope of this paper). Thus $\sigma_{N}$ is in this case given simply by $\sqrt{1000} / 1000=3.2 \%$, matching the value resulting from the simulations.

\subsection{Precision of retrieved signal intensities for two overlapping ions with perfect $m / Q$ calibration}

Repeating the process for a system with two overlapping but non-coincident Gaussian peaks leads to histograms that must be at least as broad as those shown in Fig. 1. Although the centroid positions of the two Gaussians are still fixed during both generation of the synthetic distribution and the fitting procedure, the combined Poisson-distributed uncertainties from the two peaks and the mix of information in the area of peak overlap leads to increased imprecision in the retrieved peak intensities. Figure 2 demonstrates this tendency for a pair of equally intense peaks with various different peak separations ( $\chi$, in units of HWHM). As $\chi$ increases, the predicted histogram width, $\sigma_{\mathrm{C}}$, tends towards the limiting uncertainty for an isolated ion $\sigma_{N}$, as reported by Müller et al. (2011) for a similarly posed set of simulations. For ions of equal intensity, for $\chi<1.6$ the presence of the neighbouring peak results in an appreciable increase of the normalised deviation in fitted peak intensity, consistent with the results of Müller et al. (2011).

A further example where one peak is $1 / 10$ as intense as the other is shown in Fig. S2. In this case the results are starkly different for the parent (more intense) vs. child (less intense) ions. $\sigma_{\mathrm{C}}$ of the parent ion is reduced (more precise) relative to the equal-intensity case, as the influence of the less-intense neighbouring ion is weaker, and the influence of the neighbouring peak is only appreciable for $\chi<1.2$. In contrast, $\sigma_{\mathrm{C}}$ of the child ion tends towards a value of $\sigma_{N}$ that is $\sqrt{ } 10$ higher than the equal-intensity case and is appreciably higher than this limit as soon as $\chi<1.6$.

These results are generally observed for other examples: the precision due to ion counting on an isolated ion $\sigma_{N}$ for a given system will vary as per Eq. (1), but the total precision owing to counting error, $\sigma_{\mathrm{C}}$, can be much larger than $\sigma_{N}$ when the peak separation $\chi$ is of the order of 1 , with higher separation needed for the child and less separation needed for the parent.

\subsection{Estimation of the precision of the $m / Q$ calibration}

For the constrained fitting procedures investigated in this study, which constrain a priori the positions of the fitted ions, correct determination of the $m / Q$ calibration is a prerequisite for retrieval of the ion intensities, and for the $m / Q$ it must be translated to the constrained iToF values to be used in the fits. The determination of the $m / Q$ calibration is, however, inherently subject to imprecision due to statistical effects; i.e. the fitting of the peak position of each ion (which is then used as the input of the $m / Q$ calibration) will be subject to imprecision arising from statistical effects, as per precision theory. We will estimate this imprecision in this section. Additional sources of error can be present but are not considered here. These include possible contributions from surrounding ions to the shape of the calibration ions, and incomplete knowledge of the peak shape. Additionally, the $m / Q$ calibration curve is likely to have worse precision in regions of $m / Q$ space distant from the calibration ions.

The following procedure was used to obtain a quantitative estimate of the imprecision introduced on the $m / Q$ calibration by ion counting error. We simulated the calibration procedure using synthetic data consisting of four Gaussian peaks at equally spaced iToF values $(4,8,12$ and $16 \mu \mathrm{s})$, translating to a set of corresponding known $m / Q$ values using a predefined representative set of calibration constants. The width of each peak was defined by the use of a constant resolving power 1000 , i.e. with the actual width in iToF space increasing linearly with iToF. The centroid position of the four calibrant peaks was then varied randomly according to the expected standard deviation in the peak-fitting procedure according to Eq. (2). As noted by Lee and Marshall (2000), this 


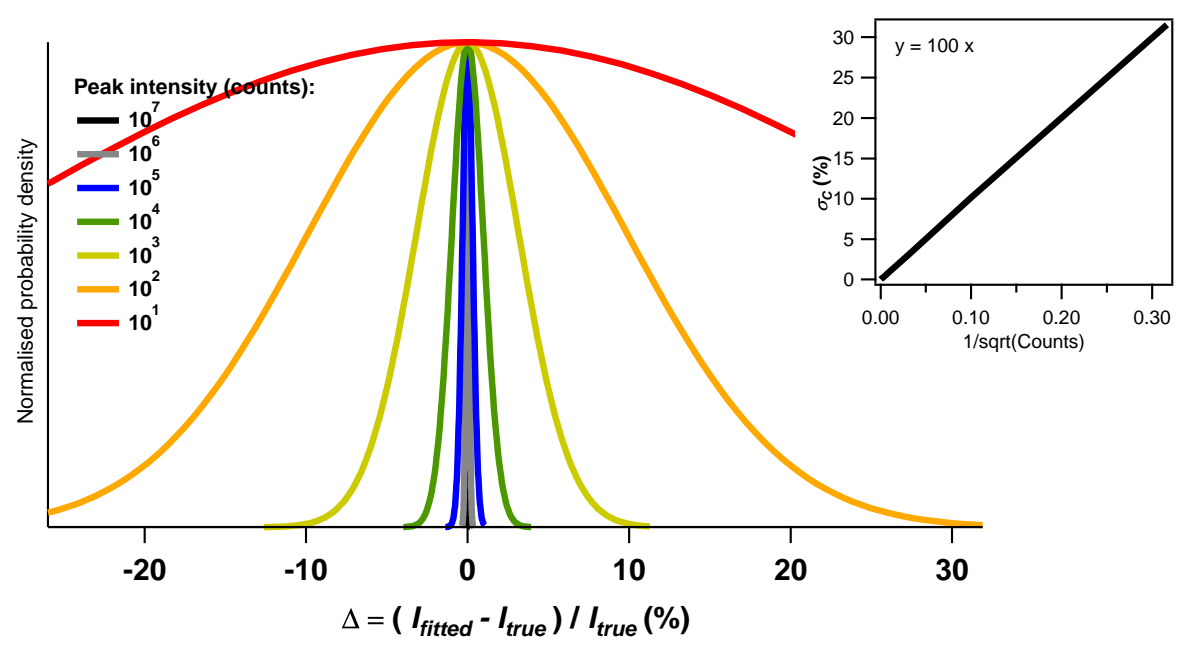

Figure 1. Histograms of the normalised deviation in fitted peak intensity, $\Delta$, for 10000 simulations of peak fitting to an isolated Gaussian ion peak degraded with counting error and centred at $2000 \mathrm{~ns}$, data-point spacing $0.2 \mathrm{~ns}$ and peak width $1 \mathrm{~ns}$. Shown inset, the linear relationship between the histogram width $\sigma_{\mathrm{C}}$, given as the standard deviation in $\Delta$ and the fitted peak intensity.

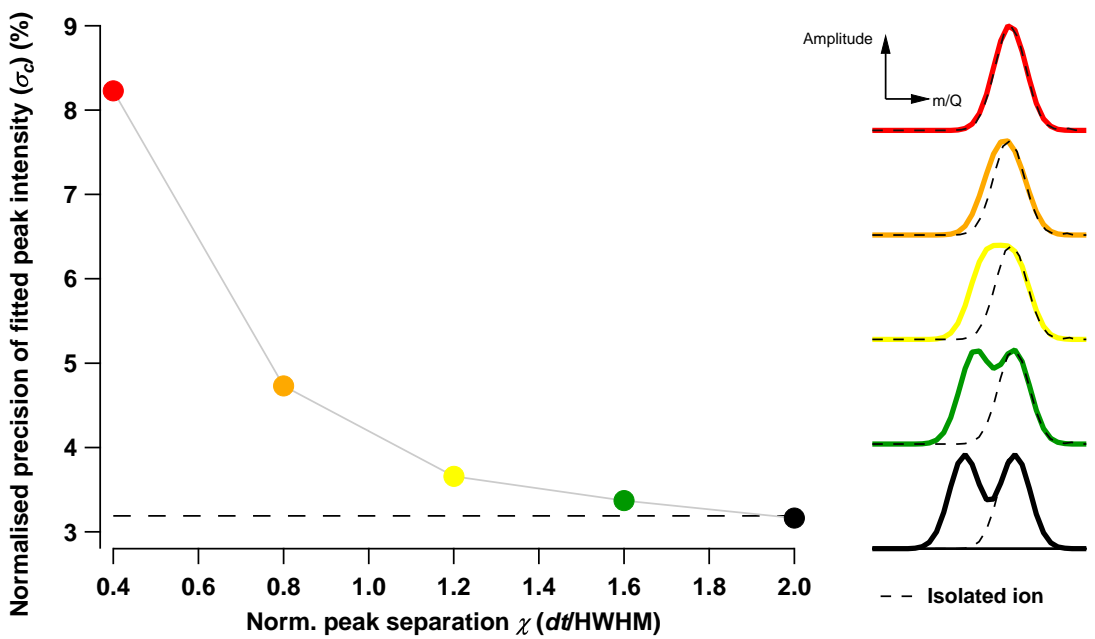

Figure 2. Relative precision in fitted peak intensity, $\sigma_{\mathrm{C}}$, for 10000 simulations of peak fitting to a pair of overlapping Gaussian peaks degraded with counting error. Peak intensity $=1000$ counts; peak width $=1 \mathrm{~ns}$; peak centre $=2000 \mathrm{~ns}$; and point spacing $=0.2 \mathrm{~ns}$. Schematics demonstrating the appearance of the measurement distributions are shown on the right, with dashed lines showing the measurement distribution of an isolated ion for comparison.

defines the theoretical upper limit to which the centroid position of the calibrant peaks in iToF space can be defined, since other possible sources of error are not accounted for. The imperfections in the fitted positions of the calibrant peaks propagate into the $m / Q$ calibration equation (a square-root relationship between iToF and $m / Q$ ). In our numerical experiment the perturbed $m / Q$ calibration was used to calculate the corresponding deviation in $\mathrm{iToF}$ for a set of $10 \mathrm{iToF}$ positions across the calibrated range, from 2 to $20 \mu$ s in $2 \mu$ s steps. It is noted that the peaks used to assess the calibration are thus not always identical to those used to define it. This was done to reflect real MS analysis, where peak fits are more often than not applied to MS regions away from the calibration points.
This procedure was repeated 10000 times to build up a set of histograms of the deviation between the known and calculated iToF values, and again repeated for resolving powers of 500, 2000 and 4000 using a constant acquisition time interval. As a longer flight path is generally required to achieve greater resolving power, the flight times of the calibrant and test peaks were also adjusted to be correspondingly shorter or longer (i.e. for resolving power 500 , using calibrants at 2 , $4,6$ and $8 \mu \mathrm{s})$.

As a result of the imprecision in the calibrant ion fits, for each iteration of the calibration procedure, each of the calibrated peak positions is subject to an error, the magnitude of which depends on the goodness of the fits to the cali- 
brant peaks and the relative positions in $m / Q$ space of the calibrants and the test peaks. Iterating the calibration procedure many times thus builds up a distribution of this error for each calibrant peak, whose standard deviation we take as the precision in the calibrated peak position. The mean normalised precision in the peak position for all the test peaks is then taken as the best estimate of the calibration precision as a whole (i.e. quantitative description of the imprecision in the calibration over all $m / Q$ ). The resulting relationships are demonstrated in Fig. 3, which shows this estimate of calibration precision for four different resolving powers and for calibrant peaks of equally intense $I_{\text {true }}$ from 10 to $10^{7}$ counts, as given on the $x$ axis.

The limiting calibration precision, where all peaks exhibit identical signal-to-noise ratios, can be less than $0.1 \mathrm{ppm}$ for high signal-to-noise situations. This unrealistic scenario exceeds the performance attainable using current mass spectrometry systems of similar resolving power, indicating that other sources of error than purely counting statistics may play a significant role in determining calibration precision. Lee and Marshall (2000) note that the relative error in $m / Q$ calibration could potentially improve by up to an order of magnitude if such sources could be eliminated. The simulations where one of the calibrant peaks suffers from poorer counting statistics (only 20 counts) also exhibit much greater calibration imprecision. Although an exhaustive investigation would be outside of the scope of this study, Fig. 3 highlights an important feature when using four ions for $m / Q$ calibration as is often typical of actual applications. If only one calibrant peak suffers from poor ion counting statistics, then even if all the others exhibit strong signal-to-noise ratios, the $m / Q$ calibration precision will be substantially degraded, by factors of 1 or 2 orders of magnitude. It is therefore important when determining the $m / Q$ calibration using some lower-intensity ions to integrate measurements for as long as possible, as long as the calibration does not drift over the averaging periods due to temperature variations or other effects.

In Sect. 3.4 we incorporate the impact of the limited precision of the $m / Q$ calibration in the overlapping peak-fitting simulations discussed in Sect. 3.2. A quantitative estimate of the precision of the $m / Q$ calibration is required. Assuming a typical case in which all calibration ions have at least 1000 counts while one ion has 20 counts, in Fig. 3, precisions of $9,5,3$ and $1.8 \mathrm{ppm}$ for the $m / Q$ calibration were used for resolving powers of 500, 1000, 2000 and 4000 respectively. Whilst larger than the limiting precisions for calibration mass spectra with high count rates, these are actually of the same order or better than values reported for real instruments of these resolving powers, both in atmospheric science (DeCarlo et al., 2006; Junninen et al., 2010; Jokinen et al., 2012) and other fields (e.g. Strittmacher et al., 2003).

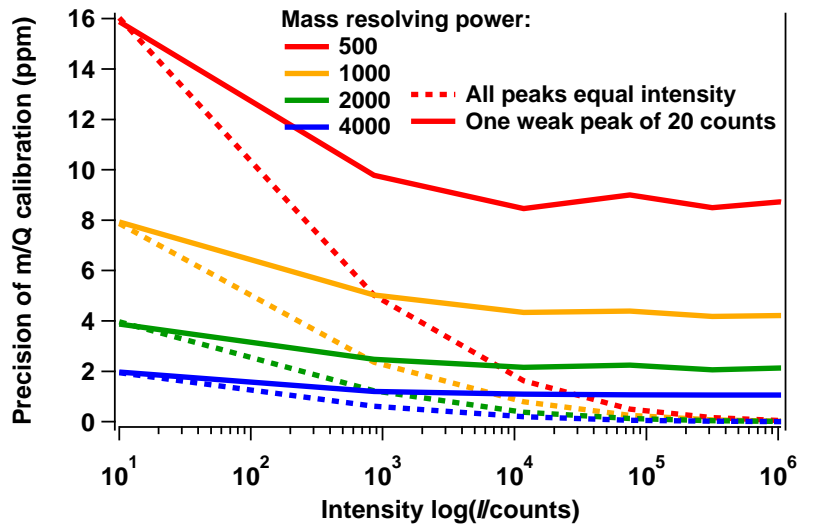

Figure 3. Mean precision in peak position arising from $m / Q$ calibration as a function of the intensity of the calibrant ions. Dashed lines: all calibrant ions of equal intensity and as given on the $x$ axis. Solid lines: as dashed, except one calibrant has peak intensity of only 20 counts.

\subsection{Precision of retrieved signal intensities in an overlapping two-ion system with realistic $m / Q$ calibration error}

We now estimate the achievable precision for the peak intensities resulting from the constrained fitting procedure, combining the precisions from counting and calibration errors in an overlapping two-ion system. As the peak positions are held fixed, uncertainties in the calibration propagate to uncertainties in the fitted intensities. Both these effects contribute to the precisions summarised in Fig. 4. These simulations use synthetic data consisting of two overlapping peaks as was demonstrated in Fig. 2 for peaks of equal intensity. However, in Fig. 4 the peak intensities are different, with a dominant "parent" peak of intensity $I_{\mathrm{p}}$ and a smaller "child" peak of intensity $I_{\mathrm{c}}$, with $R_{I}=I_{\mathrm{p}} / I_{\mathrm{c}}=2$. The precisions in the normalised deviation in retrieved peak intensity of the parent and child peaks, $\sigma_{\mathrm{p}}$ and $\sigma_{\mathrm{c}}$ respectively, are shown. The values of $\sigma_{N}$ (the counting-error limit) for an isolated ion are also shown for comparison.

Three regimes are apparent in this plot:

1. For large $\chi$ (well-separated peaks), the precision in the normalised deviation in retrieved peak intensity, $\sigma_{I}$, for both peaks is limited by counting error and tends towards the $\sigma_{N}$ values for isolated ions; i.e. as the ion separation increases both ions are retrieved with similar precision to individual isolated ions. This region we term the "counting-error regime". Note that for wellseparated peaks the imprecision in the $m / Q$ calibration at the realistic levels used here is small relative to the counting error and does not result in an increase in the precision above that imposed by counting statistics.

2. For smaller values of $\chi(<\sim 3$, overlapping peaks $)$ and higher signal levels, $\sigma_{I}$ is observed to increase rapidly 

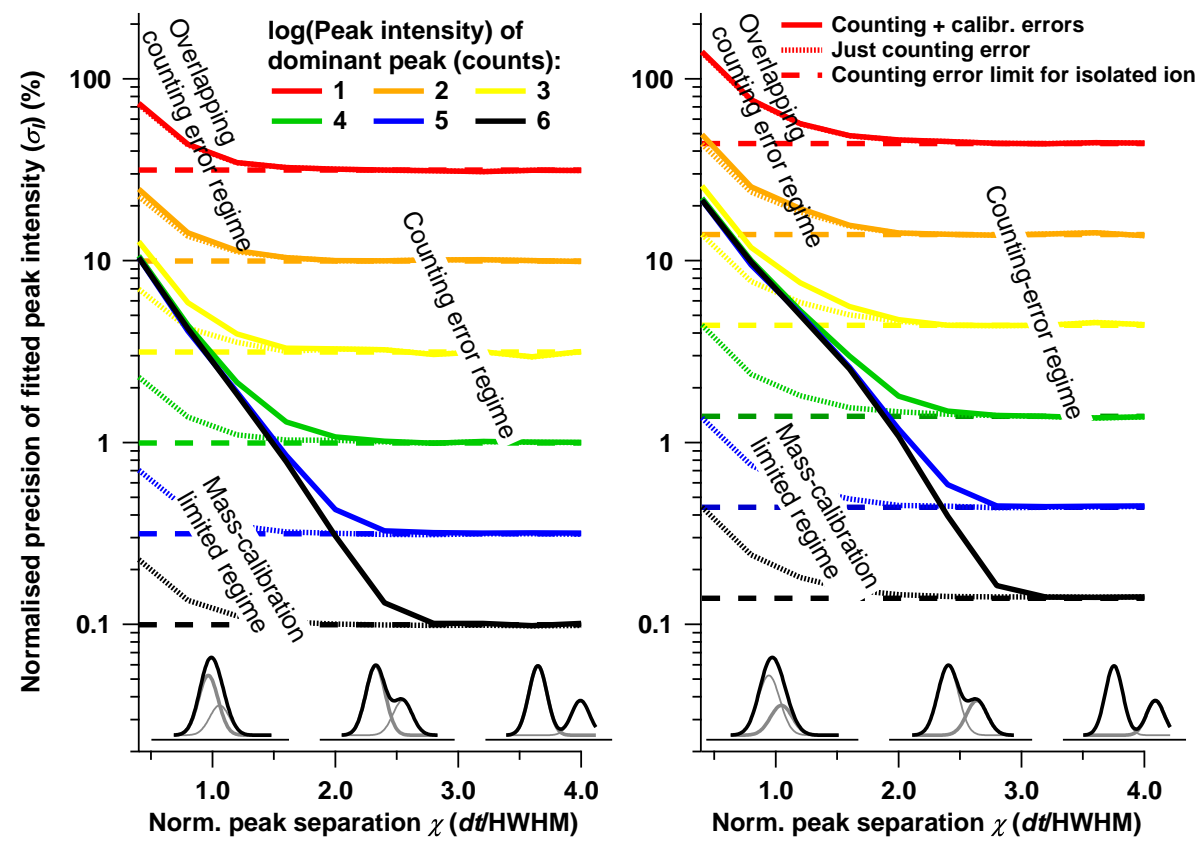

Figure 4. Precision on fitted peak intensity, $\sigma_{I}$, as a function of peak separation for an overlapping ion pair. Colours: peak intensity of the dominant ion (left); the weaker ion (right) is half as intense. The dominant peak is centred at $2000 \mathrm{~ns}$; both have a width of $1 \mathrm{~ns}$, and the data-point spacing is $0.2 \mathrm{~ns}$.

above the counting-error limit $\sigma_{N}$. This region we term the " $m / Q$ calibration-limited regime".

3. For the special case of a low signal-to-noise ratio and small $\chi(<\sim 2$, strongly overlapping peaks $)$, the overlapping nature of the peak distribution increases $\sigma_{I}$ above $\sigma_{N}$ in a manner which is dominated by the overlapping peak effect (as in Sect. 3.2) and not affected by the $m / Q$ calibration error. As discussed above, the effect is stronger for the weaker child peak than it is for the stronger parent peak. This region we term the "overlapping counting-error regime", and $\sigma_{I} \sim \sigma_{\mathrm{C}}$.

In summary, for low signal-to-noise ion pairs (in our particular examples for peak intensities of order 100 counts), the influence of the $m / Q$ calibration imprecision on $\sigma_{I}$ is negligible, and the counting and overlapping effects discussed in Sects. 3.1 and 3.2 dominate. Indeed, in the absence of $m / Q$ calibration imprecision, $\sigma_{\mathrm{C}}(\sigma$ arising only from counting error) for a given separation $\chi$ increases for both ions simply as the square root of the respective peak's intensity. The same relationship is, as expected, also true for $\sigma_{I}$ in the countingerror-dominated regime. However, the introduction of $m / Q$ calibration imprecision in the simulations leads to a higher $\sigma_{I}$ for small values of $\chi$ that sometimes greatly exceeds (e.g. $>10-100$ times larger) the precision introduced by counting error for overlapping ions alone.

The different regimes introduced in Fig. 4 can be visualised for a range of $I_{\mathrm{p}}$ and fixed $R_{I}=8$ by representing $\sigma_{I}$ in an image plot as shown in Fig. 5 (top). The transition be- tween the two regimes at $\chi=\chi_{d}$ is shown superimposed as the black line over the image. To enable quantitative determination of this parameter, we define $\chi_{d}$ as the point at which $\sigma_{I}=1.05 \cdot \sigma$ at $\chi=4 \sim \sigma_{\mathrm{C}}$. For $\chi<\chi_{d}$, the areas of similar $\sigma_{I}$ appear predominantly as vertically orientated rectangles. This shows how integrating to higher ion counts does not change $\sigma_{I}$ in the calibration-limited regime. The only case for $\chi<\chi_{d}$ where integrating improves $\sigma_{I}$ is for the overlapping counting-error regime (small signal-to-noise ratio and small $\chi$ ), seen by the small change in $\sigma_{I}$ just above and left of the $\chi=\chi_{d}$ line for $\chi<\sim 1$. In contrast, for $\chi>\chi_{d}$, the areas of similar $\sigma_{I}$ appear as horizontally orientated rectangles. This is the counting-error regime, and integrating to higher ion counts will improve $\sigma_{I}$. So long as $\chi>\chi_{d}$, peak separation is unimportant for determination of $\sigma_{I}$ for both the parent and child peaks.

\subsection{Parameterisation of the intensity precision for overlapping ions with $m / Q$ calibration error: independent parameters}

Since our simulations are complex and time consuming, a parameterisation of the value of $\sigma_{B}$, the limiting precision due to $m / Q$ calibration imprecision, is desirable in assessing fitting precision. We note that, in the case of high ion counts, $\sigma_{B} \sim \sigma_{I}$, for uncertainty arising from counting error is negligible in comparison to that introduced by calibration imprecision. Furthermore, from Fig. 4, as $\chi$ is decreased, the limiting values of $\sigma_{I}$ for different peak intensities all fall 


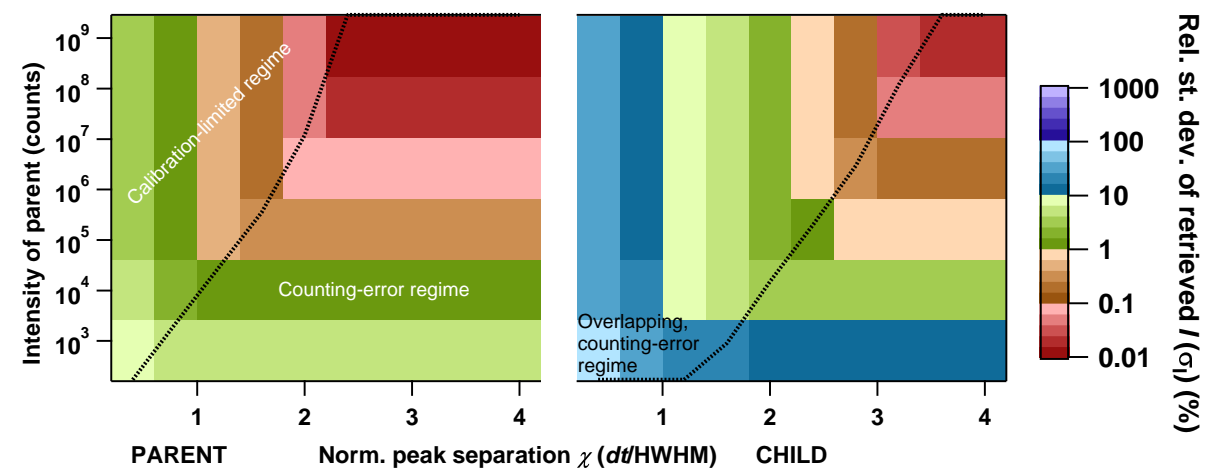

Figure 5. Actual precision in the normalised fitted peak intensities $\sigma_{I}$ for an overlapping ion pair. The stronger (parent) peak is 8 times as intense as the weaker (child) peak. The precision is represented by the colour scale and shown for a range of peak intensities and separations. The black lines denote the peak separation at or below which an ion would be considered to be in the calibration-limited regime.

along the same line (with the exception of the overlapping counting-error regime). This implies that $\sigma_{B}$ is insensitive to peak intensity. Further simulations were thus conducted to assess the sensitivity of $\sigma_{B}$ to various parameters in the fit, altering one parameter at a time and holding the peak intensity ratio $R_{I}$ fixed at 2 . These results are reduced to scalar values in Table 1 by reporting the value of $\sigma_{I}$ for the child peak at $\chi=1.0$ for the default, and altered inputs. By considering only results at high ion counts, we assume $\sigma_{B} \sim \sigma_{I}$ and thus effectively report $\sigma_{B}$ at $\chi=1.0$.

Table 1 demonstrates that $\sigma_{B}$ is insensitive to changes in the spectrometer resolving power, data-acquisition rate (point spacing) and peak intensity. Only increasing the estimate of imprecision in the $m / Q$ calibration had an appreciable impact on the simulations. Tripling the calibration imprecision roughly doubled $\sigma_{B}$. A conservative estimate of $\sigma_{B}$ could therefore be up to twice the values reported in this study (which we consider the best-case scenario) but is almost certainly not going to be larger than that for a wellcalibrated system.

Considered together, these observations imply that the precision owing to calibration imprecision, $\sigma_{B}$, can be considered a function of only $\chi$ and $R_{I}$ and is independent of $W, \delta_{\mathrm{t}}$ and $I_{\mathrm{p}}$.

\subsection{Parameterisation of the intensity precision for overlapping ions with $m / Q$ calibration error: parameterisation}

Given the weak dependence of $\sigma_{B}$ upon $I_{\mathrm{p}}, W$ and $\delta_{\mathrm{t}}$ shown in the previous section, we thus propose that $\sigma_{B}$ for the child peak in the resolving power range considered in this study $(<4000)$ can be empirically parameterised by fitting a polynomial in $\log \sigma$-space to the $m / Q$ calibration-limited regime section of the results for high signal-to-noise ratios (Fig. S3). In this region $\sigma_{I} \sim \sigma_{B}$, i.e. the imprecision arising from calibration effects, dominates and counting error can be consid- ered negligible. This leads to the relationships

$\sigma_{B}($ child $)=\frac{R_{I}}{0.6}\left[10^{\left(0.6-0.41(\chi-0.4)-0.2(\chi-0.4)^{2}\right)}\right]$,
$\sigma_{B}($ parent $)=10^{\left(0.6-0.41(\chi-0.4)-0.2(\chi-0.4)^{2}\right)}$.

These equations are derived from simulations using the estimated best-achievable calibration precision. Following Table 1, a more conservative approach might choose values up to double those calculated using these formulae, according to the expected imprecision in the $m / Q$ calibration. We note that, although the relationships are independent of resolving power, intensity and sample interval as shown in Table 1, for application to the child peak it does it does depend linearly on the intensity ratio $R_{I}$.

Given that $\sigma_{N}$ is independent of $\chi$ and easily calculated from $W, \delta_{\mathrm{t}}, I_{\mathrm{p}}$ and $R_{I}$ using Eq. (1), it is possible to describe the minimum estimated precision on $I_{\mathrm{c}}$ as the larger of the calculated $\sigma_{N}$ and parameterised $\sigma_{B}$. The two lines cross at $\chi_{d}$, the value of $\chi$ at which $\sigma_{I}$ is observed to diverge from the counting-error limit of an isolated peak. For $\chi<\chi_{d}, \sigma_{I}$ can be estimated from the parameterisation of $\sigma_{B}$, whereas for $\chi>\chi_{d}, \sigma_{I}$ can be calculated directly and is equal to $\sigma_{N}$.

This estimate of precision, whilst predominantly generally applicable for the resolving power range considered in this study, does break down in the overlapping counting-error regime (low signal-to-noise ratio and small peak separation). This is apparent when studying Fig. 4, which demonstrates that a maximum underestimate (at very low $\chi$ ) in $\sigma_{I}$ of 2 and 5 would be expected for $I_{\mathrm{p}}=10$ and 100 counts respectively. The parameterisation described here thus describes the bestcase scenario and should be regarded as an estimate of the lower limit of $\sigma_{I}$ expected for a given system.

An example of the application of these results is given in Fig. 6. Lines of constant $\sigma_{B}$ are estimated for a range of peak intensity ratios $R_{I}$ and separation $\chi$ using the parameterisation described above. These are plotted together with markers representing $R_{I}$ and $\chi$ for the less-intense (child) peak of all pairs of peaks in a fixed- $m / Q$ list applied in the analysis of 
Table 1. Changes in precision in fitted intensity of the child peak when altering properties of the fitting procedure and its input distributions, whilst holding the peak intensity ratio constant at 2 .

\begin{tabular}{|c|c|c|c|}
\hline \multirow{2}{*}{$\begin{array}{l}\text { Parameter adjusted } \\
\text { Calibration } \\
\text { imprecision }\end{array}$} & \multirow{2}{*}{$\begin{array}{l}\sigma_{I} \text { at } \chi=1.0 \\
\text { for default value } \\
35 \% \text { at } 5 \mathrm{ppm}\end{array}$} & \multicolumn{2}{|c|}{$\begin{array}{l}\sigma_{I} \text { at } \chi=1.0 \\
\text { for adjusted value }\end{array}$} \\
\hline & & $64 \%$ at $10 \mathrm{ppm}$ & $82 \%$ at $15 \mathrm{ppm}$ \\
\hline Resolving power & $35 \%$ at 1000 & $32 \%$ at 500 & $41 \%$ at 2000 \\
\hline Intensity & $35 \%$ at $10^{4}$ counts & $35 \%$ at $10^{5}$ counts & $35 \%$ at $10^{7}$ counts \\
\hline Sample interval & $35 \%$ at $1 \mathrm{~ns}$ & $34 \%$ at $0.5 \mathrm{~ns}$ & $35 \%$ at $2 \mathrm{~ns}$ \\
\hline
\end{tabular}

real experimental data for the CalNex data set (Hayes et al., 2013). $\chi$ can be calculated directly from the list; $R_{I}$ is however calculated from the fit results for a representative average spectrum taken from the campaign data set and is therefore subject to the same sources of imprecision discussed in this study. The influence of additional overlapping peaks is also ignored. Nonetheless, representation of the fitted peak pairs in this space provides a useful overview of the reliability of the fitted intensities. $\sigma_{N}$ is represented by colouring the data points similarly to the predicted lines of precision from the parameterisation of $\sigma_{B}$. The estimated precision is thus the larger of the two. In this example, the peaks fitted mostly exhibit good separation, and counting error is the limiting factor. It is noted that the region for $\chi<0.4$ was not explicitly studied as part of this work, and the lines of estimated precision would have no meaning as $\chi$ approached zero. This region is thus not shown on the plot.

The optimal experimental setup for a given ion pair is where $\chi=\chi_{d}$, for then intensity imprecision due to $m / Q$ calibration is negligible, but the number of points measured across the peak is maximised.

To demonstrate the application of the parameterisation to a problem relevant to the atmospheric-science community that provided motivation for this study, we take three commonly observed ions in $\mathrm{HR}-\mathrm{ToF}-\mathrm{AMS}$ spectra as an example: $\mathrm{C}_{2} \mathrm{H}_{3} \mathrm{O}^{+}(m / Q=43.015 \mathrm{Th}), \mathrm{C}_{3} \mathrm{H}_{7}^{+}$(43.054) and $\mathrm{C}_{2} \mathrm{H}_{5} \mathrm{~N}^{+}$ (43.043). Müller et al. (2011) concluded that $\mathrm{C}_{2} \mathrm{H}_{3} \mathrm{O}^{+}$and $\mathrm{C}_{2} \mathrm{H}_{5} \mathrm{~N}^{+}$were distinguishable applying fits to a spectrum with mass resolving power 1000 (i.e. $\chi=1.3$ ); from our parameterisation, a constrained fit to these peak positions would retrieve the peak intensity to within a precision of $5 \%$ only for $R_{I}<6$ (assuming sufficient counting statistics), even though the presence of both the peaks may be evident in the spectrum for higher $R_{I}$ (the overlapping peaks for this scenario are exemplified in Fig. S4). For the closely separated $\mathrm{C}_{3} \mathrm{H}_{7}^{+}$and $\mathrm{C}_{2} \mathrm{H}_{5} \mathrm{~N}^{+}$ions, Farmer et al. (2010) concluded that a mass resolving power of 5000 would be desirable; this would result in $\chi=2.5$ and thus a $25 \%$ precision on $I_{\mathrm{c}}$ for $R_{I}$ as high as 400 . Up to $1 \%$ precision would be achievable for $R_{I} \sim 15$ (the overlapping peaks for this scenario are exemplified in Fig. S5). With half this resolving power the same precision can only be expected for $R_{I}$ less than $\sim 20$.

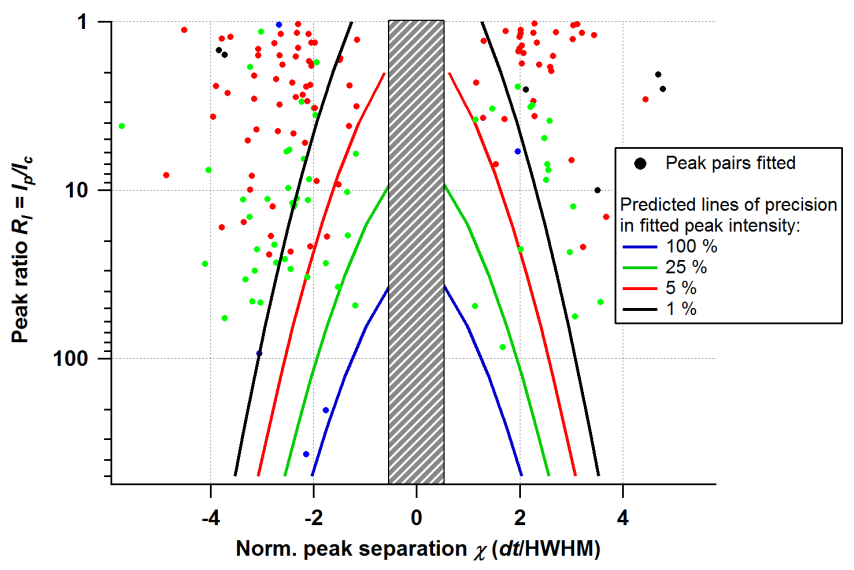

Figure 6. Ratio of fitted peak intensities to true peak separation for the smaller ion from all pairs of ions used in analysis of the CalNex field campaign data from a high-resolution aerosol mass spectrometer (Hayes et al., 2013). Superimposed are lines of constant estimated precision on fitted peak intensity. The data points are coloured according to the precision in fitted peak intensity expected from counting statistics. The data-point spacing is $0.2 \mathrm{~ns}$, and peak width is $1 \mathrm{~ns}$.

\section{Conclusions}

A simple statistical simulation-based approach has been used to demonstrate the precision to which the intensities for a pair of overlapping mass spectral peaks can be ascertained using least-squares multi-peak fitting. Synthetic measurement distributions containing imprecisions from counting statistics and $m / Q$ calibration were used in a fitting process where peak centres and widths were constrained. Three principal regimes appear: (1) the counting-limited regime: for wellseparated and/or dominantly abundant ions, the best precision on the retrieved peak intensity follows the theoretical limit imposed by counting statistics; (2) the $m / Q$ calibrationlimited regime: for closely separated ions, the influence of the imprecision in the $m / Q$ calibration dominates and the achievable precision on peak intensity degrades rapidly; and (3) the overlapping counting-error regime: at a low signal-tonoise ratio, the imprecision arising from counting error still dominates and the imprecision in the $m / Q$ calibration makes 
no additional impact. The transition between the counting error and calibration-limited regime depends on the intensity of the peaks but is only weakly sensitive to their absolute width. Thus a general parameterisation can be developed which describes the lower limits in peak separation and intensity that are required for a given precision in the fits. Except for cases of a poor signal-to-noise ratio $(<\sim 100$ counts $)$, integration to longer measurement timescales for an ion in the calibration-limited regime cannot improve the precision with which its peak intensity can be retrieved; in order to move the ion into the counting-error regime, higher resolving power would be required.
The results are demonstrated as applied to a typical instrumental setup employed in atmospheric science but make no assumptions about ionisation or spectrometer type and can thus be generally applied. Further investigations to include the effect of imprecisions in peak width, non-Gaussian peak shape and systems with greater than two ion peaks should form the basis of future work. 


\section{Appendix A: Glossary}

\begin{tabular}{|c|c|}
\hline$\chi$ & $\begin{array}{l}\text { Peak separation normalised to half-width at } \\
\text { half-maximum (HWHM) }\end{array}$ \\
\hline$\chi_{d}$ & $\begin{array}{l}\text { Peak separation below which ion would be considered } \\
\text { to be in the } m / Q \text { calibration-limited regime }\end{array}$ \\
\hline$I$ & Peak intensity (in number of ion counts) \\
\hline$I_{\text {true }}$ & True peak intensity \\
\hline$I_{\text {fitted }}$ & Fitted peak intensity \\
\hline$\Delta$ & $\begin{array}{l}\text { Normalised deviation in fitted peak intensity } \\
\left(I_{\text {fitted }}-I_{\text {true }}\right) / I_{\text {true }}\end{array}$ \\
\hline$I_{\mathrm{p}}$ & $\begin{array}{l}\text { Peak intensity of parent (dominant) peak in an } \\
\text { overlapping pair }\end{array}$ \\
\hline$I_{\mathrm{c}}$ & $\begin{array}{l}\text { Peak intensity of child (non-dominant) peak in an } \\
\text { overlapping pair }\end{array}$ \\
\hline$R_{I}$ & $\begin{array}{l}\text { Ratio of parent-to-child intensities for an overlapping } \\
\text { ion pair }\left(I_{\mathrm{p}} / I_{\mathrm{c}}\right)\end{array}$ \\
\hline$\sigma_{I}$ & $\begin{array}{l}\text { Normalized precision of the fitted peak intensity owing } \\
\text { to all sources of error }(\%)\end{array}$ \\
\hline$\sigma_{C}$ & $\begin{array}{l}\text { Normalized precision of the fitted peak intensity owing } \\
\text { to counting error only }(\%)\end{array}$ \\
\hline$\sigma_{N}$ & $\begin{array}{l}\text { Minimum achievable } \sigma_{I} \text { owing to counting error for } \\
\text { an isolated ion }(\%)\end{array}$ \\
\hline$\sigma_{B}$ & $\begin{array}{l}\text { Minimum achievable } \sigma_{I} \text { owing to } m / Q \text { calibration } \\
\text { imprecision }(\%)\end{array}$ \\
\hline$S_{\mathrm{A}}$ & Standard deviation in fitted peak amplitude \\
\hline$S_{\mathrm{t}}$ & Standard deviation in fitted centroid iToF position \\
\hline$W$ & Peak width (ns) \\
\hline$\delta_{t}$ & Data-point spacing (ns) \\
\hline $\mathrm{d} t$ & $\begin{array}{l}\text { Separation of the known peak positions in } \\
\text { time-of-flight space }\end{array}$ \\
\hline $\mathrm{iToF}$ & Ion time of flight \\
\hline "Imprecision" & $\begin{array}{l}\text { is qualitatively used to describe the repeatability of } \\
\text { results }\end{array}$ \\
\hline "Precision" & $\begin{array}{l}\text { is quantitatively used to describe the standard deviation } \\
\text { of the results obtained under repeatability conditions }\end{array}$ \\
\hline
\end{tabular}




\section{The Supplement related to this article is available online at doi:10.5194/amt-8-2333-2015-supplement.}

Acknowledgements. This work was partially supported by NSF AGS-1243354 and AGS-1360834, NOAA NA13OAR4310063, NASA NNX12AC03G, and DOE (BER, ASR) DE-SC0011105. We thank Marc Gonin for useful discussions on this topic. We thank D. Sueper for providing the CalNex data.

Edited by: F. Stroh

\section{References}

Bantscheff, M., Schirle, M., Sweetman, G., Rick, J., and Kuster, B.: Quantitative mass spectrometry in proteomics: a critical review, Anal. Bioanal. Chem., 389, 1017-1031, doi:10.1007/s00216007-1486-6, 2007.

Bantscheff, M., Lemeer, S., Savitski, M. M., and Kuster, B.: Quantitative mass spectrometry in proteomics: critical review update from 2007 to the present. Anal. Bioanal. Biochem., 404, 939965, doi:10.1007/s00216-012-6203-4, 2012.

Blom, K. F.: Utility of Peak Shape Analyses in Determining Unresolved Interferences in Exact Mass Measurements at Low Resolution, J. Am. Soc. Mass Spectrom., 9, 789-798, 1998.

Cappellin, L., Biasioli, F., Fabris, A., Schuhfried, E., Soukoulis, C., Märk, T. D., and Gasperi, F.: Improved mass accuracy in PTR-TOF-MS: Another step towards better compound identification in PTR-MS, Int. J. Mass Spectrom., 290, 60-63, doi:10.1016/j.ijms.2009.11.007, 2009.

Cappellin, L., Biasioli, F., Granittoc, P. M., Schuhfried, E., Soukoulis, C., Costa, F., Märk, D. T., and Gasperi, F.: On data analysis in PTR-TOF-MS: From raw spectra to data mining. Sensors and Actuators B, 155, 183-190, doi:10.1016/j.snb.2010.11.044, 2011.

DeCarlo, P. F., Kimmel, J. R., Trimborn, A., Northway, M. J., Jayne, J. T., Aiken, A. C., Gonin, M., Fuhrer, K., Horvath, T., Docherty, K., Worsnop, D. R., and Jimenez, J. L.: field-deployable, highresolution, Time-of-Flight Aerosol Mass Spectrometer, Anal. Chem., 78, 8281-8289, 2006.

Farmer, D. K. and Jimenez, J. L.: Real-time atmospheric chemistry field instrumentation, Anal. Chem., 82, 7879-7884, 2010.

Farmer, D. K., Matsunaga, A., Docherty, K. S., Surratt, J. D., Seinfeld, J. H., Ziemann, P. J., and Jimenez, J. L.: Response of an aerosol mass spectrometer to organonitrates and organosulfates and implications for atmospheric chemistry, PNAS, 107, 66706675, 2010.

Fraga, C. G. and Corley, C. A.: The chemometric resolution and quantification of overlapped peaks form comprehensive twodimensional liquid chromatography, J. Chromatography A, 1096, 40-49, 2005.

Gardner, R. P., Ai, X., Peeples, C. R., Wang, J., Lee, K., Peeples, J. L., and Calderon, A.: Use of an iterative convolution approach for qualitative and quantitative peak analysis in low 15 resolution gamma-ray spectra, Symposium on Radiation Measurements and Applications (SORMA) XII 2010, Nucl. Instrum. Meth. A, 652, 544-549, 2011.
Haimi, P., Uphoff, A., Hermansson, M., and Somerharju, P.: Software Tools for Analysis of Mass Spectrometric Lipidome Data, Anal. Chem., 78, 8324-8331, doi:10.1021/ac061390w, 2006.

Hammed, M. A., Gray, P. W., Naboulsi, A. H., and MacMahon, T. H.: Analytical peak fitting for gamma-ray spectrum analysis with Ge detectors. Nuclear Instruments and Methods in Physics Research Section A: Accelerators, Spectrometers, Detectors and Associated Equipment, 334, 543-550, 1993.

Hayes, P. L., Ortega, A. M., Cubison, M. J., Froyd, K. D., Zhao, Y., Cliff, S. S., Hu, W. W., Toohey, D. W., Flynn, J. H., Lefer, B. L., Grossberg, N., Alvarez, S., Rappenglück, B., Taylor, J. W., Allan, J. D., Holloway, J. S., Gilman, J. B., Kuster, W. C., de Gouw, J. A., Massoli, P., Zhang, X., Liu, J., Weber, R. J., Corrigan, A. L., Russell, L. M., Isaacman, G., Worton, D. W., Kreisberg, N. M., Goldstein, A. H., Thalman, R., Waxman, E. M., Volkamer, R., Lin, Y. H., Surratt, J. D., Kleindienst, T. E., Offenberg, J. H., Dusanter, S., Griffith, S., Stevens, P. S., Brioude, J., Angevine, W. M., and Jimenez, J. L.: Organic aerosol composition and sources in Pasadena, California, during the 2010 CalNex campaign, J. Geophys. Res.-Atmos., 118, 9233-9257, 2013.

Hilmer, J. K. and Bothner, B.: Physical signal modulation of timeof-flight mass analyzers increases precision and decreases noise, Rapid Commun. Mass Spectrom., 25, 795-805, 2001.

Horn, D. M., Zubarev, R. A., and McLafferty, F. W.: Automated reduction and interpretation of high resolution electrospray mass spectra of large molecules, J. Am. Soc. Mass Spectrom., 11, 320 332, doi:10.1016/S1044-0305(99)00157-9, 2000.

House, L. L., Clyde, M. A., and Wolpert, R. L.: Baysian nonparametric models for peak identification in MALDI-TOF mass spectroscopy, Ann. Appl. Stat., 5, 1488-1511, doi:10.1214/10AOAS450, 2011.

Jaitly, N., Mayampurath, A., Littlefield, K., Adkins, J. N., Anderson, G. A., and Smith, R. D.: Decon2LS: An open-source software package for automated processing and visualization of high resolution mass spectrometry data, BMC Bioinformatics, 10, 87, doi:10.1186/1471-2105-10-87, 2009.

Jokinen, T., Sipilä, M., Junninen, H., Ehn, M., Lönn, G., Hakala, J., Petäjä, T., Mauldin III, R. L., Kulmala, M., and Worsnop, D. R.: Atmospheric sulphuric acid and neutral cluster measurements using CI-APi-TOF, Atmos. Chem. Phys., 12, 4117-4125, doi:10.5194/acp-12-4117-2012, 2012.

Junninen, H., Ehn, M., Petäjä, T., Luosujärvi, L., Kotiaho, T., Kostiainen, R., Rohner, U., Gonin, M., Fuhrer, K., Kulmala, M., and Worsnop, D. R.: A high-resolution mass spectrometer to measure atmospheric ion composition, Atmos. Meas. Tech., 3, 10391053, doi:10.5194/amt-3-1039-2010, 2010.

Kilgour, D. P. A., Mackay, C. L., Langridge-Smith, P. R. R., and O'Connor, P. B.:Appropriate Degree of Trust: Deriving Confidence Metrics for Automatic Peak Assignment in High-Resolution Mass Spectrometry, Anal. Chem., 7431-7435, doi:10.1021/ac301339d, 2012.

Krupcik, J., Mydlova, J., Spanik, I., Tienpont, B., and Sandra, P.: Computerized separation of chromatographically unresolved peaks. J. Chromotography A, 1084, 80-89, doi:10.1016/j.chroma.2004.09.073, 2005.

Laeven, J. M. and Smit, H. C.: Optimal peak area determination in the presence of noise, Anal. Chim. Acta, 176, 77-104, 1985.

Link, A. J., Eng, J., Schieltz, D. M., Carmack, E., Mize, G. J., Morris, D. R., Garvik, B. M., and Yates, J. R.: Direct analysis of 
protein complexes using mass spectrometry, Nature Biotechnol., 17, 676-682, 1999.

Lee, H.-N. and Marshall, A. G.: Theoretical Maximal Precision for Mass-to-Charge Ratio, Amplitude, and Width Measurements in Ion-Counting Mass Analyzers, Anal. Chem., 72, 2256-2260, 2000.

Meija, J. and Caruso, J. A.: Deconvolution of Isobaric Interferences in Mass Spectra, J. Am. Soc. Mass Spectrom., 15, 654-658, 2004.

Mirgorodskaya, O. A., Kozmin, Y. P., Titov, M. I., Körner, R., Sönksen, C. P., and Roepstorff P.: Quantitation of peptides and proteins by matrix-assisted laser desorption/ionization mass spectrometry using ${ }^{18} \mathrm{O}$-labeled internal standards, Rapid Commun. Mass Spectrom., 14, 1226-1232, 2000.

Müller, M., George, C., and D'Anna, B.: Enhanced Spectral Analysis of C-TOF Aerosol Mass Spectrometer Data: Iterative Residual Analysis and Cumulative Peak Fitting. Int. J. Mass Spectrom., 306, 1-8, doi:10.1016/j.ijms.2011.04.007, 2011.

Strittmatter, E. F., Rodriguez, N., and Smith, R. D.: High Mass Measurement Accuracy Determination for Proteomics Using Multivariate Regression Fitting: Application to Electrospray Ionization Time-Of-Flight Mass Spectrometry, Anal. Chem., 75, 460468, 2003.
Sun, Y. T., Zhang, J. Q., Braga-Neto, U., and Dougherty, E. R.: BPDA - A Bayesian peptide detection algorithm for mass spectrometry, BMC Bioinformatics, 11, 490, doi:10.1186/14712105-11-490, 2010.

Titzmann, T., Grausb, M., Müller, M., Hansel, A., and Ostermann, A.: Improved peak analysis of signals based on counting systems: Illustrated for proton-transfer-reaction time-offlight mass spectrometry, Int. J. Mass Spectrom., 295, 72-77, doi:10.1016/j.ijms.2010.07.009, 2010.

Uher, J., Roach, G., and Tickner, J.: Peak fitting and identification software library for high resolution gamma-ray spectra, Nucl. Instrum. Meth. A, 619, 457-459, doi:10.1016/j.nima.2009.12.086, 2010.

Yatavelli, R. L. N., Lopez-Hilfiker, F., Wargo, J. D., Kimmel, J. R., Cubison, M. J., Bertram, T. H., Jimenez, J. L., Gonin, M., Worsnop, D. R., and Thornton, J. A.: A Chemical Ionization High-Resolution Time-of-Flight Mass Spectrometer Coupled to a Micro Orifice Volatilization Impactor (MOVI-HR-ToF-CIMS) for Analysis of Gas and Particle-Phase Organic Species, Aerosol Sci. Technol., 46, 1313-1327, 2012.

Yu, T. and Peng, H.: Quantification and deconvolution of asymmetric LC-MS peaks using the bi-Gaussian mixture model and statistical model selection, BMC Bioinformatics, 11, 11:559-559, doi:10.1186/1471-2105-11-559, 2010. 